\title{
Death Inc.
}
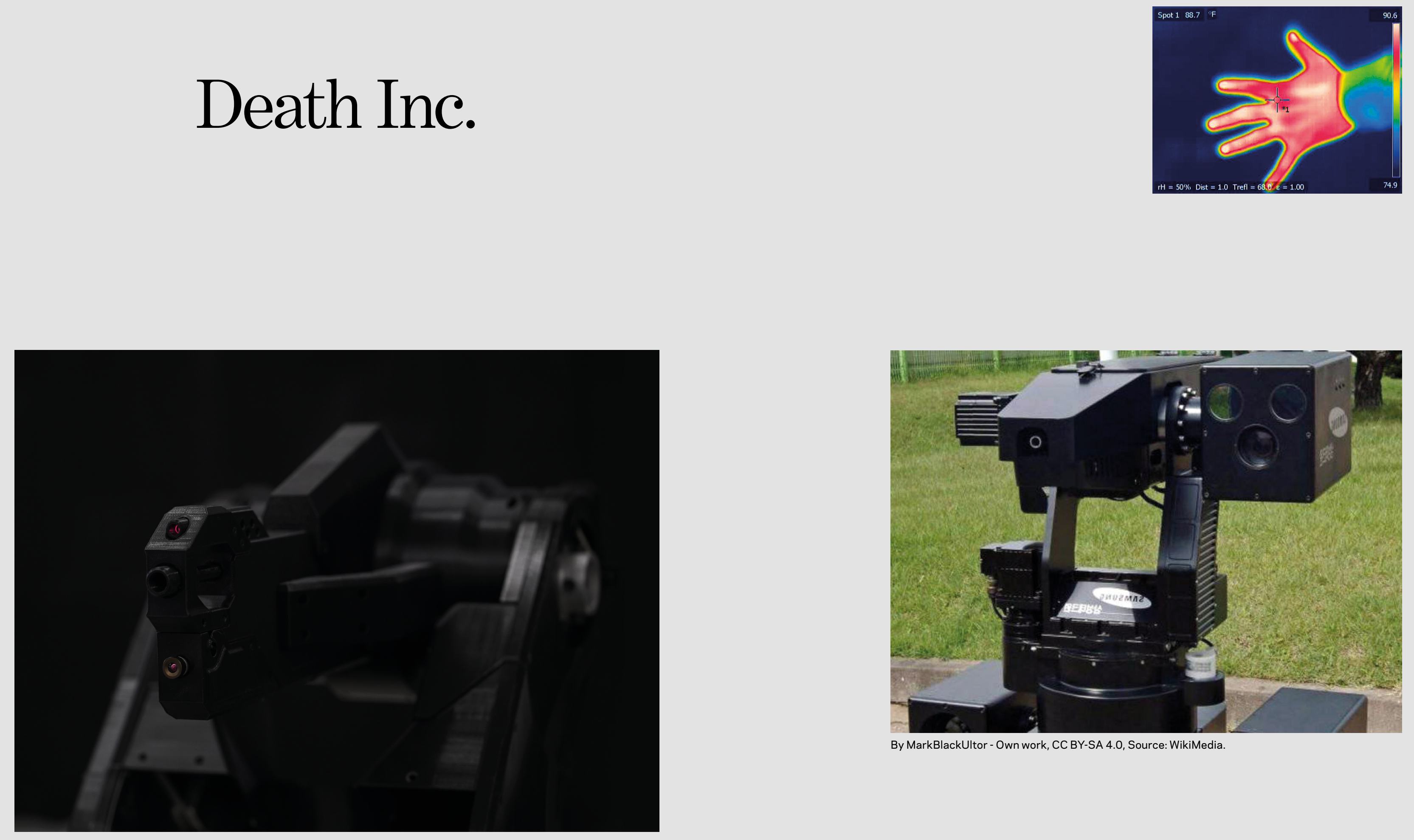

In March 23, 2018, the exhibition "Design Does"
or better and for tonsen Disseny de Barcelona (Design Museum). The exhibidiscussion about the roles and responsibilities of design in our society.
It featured open-ended questions, with neither a titions such as: should With this question Design Does" presented the piece Death Inc., a reflection on design applied to
the arms industry. The exploration of a new style of killing that arises from the incorporation of image recognition technologies to military robots. decision. Death Inc. Project arose from the scientific Principle of Discrimination" (Guersenzzaig, 2018) which explores the moral and ethical consequences (a) the demilitarized zone of the South Korea-North Korea border. This model, considered the first of ts kind, marks the start of a new human-machine interaction in which the machine has been designed
to be able to select and shoot human targets with-
out requiring a person to authorize the operation. By MarkBlackUltor - Own work, CC BY-SA 4.O, Source: WikiMedia.

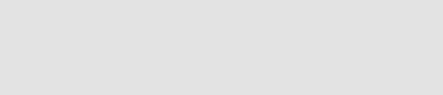


Autonomous weapons without human control (Hu
mans Out-Of-The-Loop) are here to stay. Despit the official pledge on futureoflife.org against Lethal
Autonomous Weapons, or LAWs, signed by highctor such as Google Deep Mind and Elon Musk. LAW investments and popularity are increasing yearly Scharre 2018)

the atomic bomb once upon a time, will revolutitionize the way we understand war. Today, the SGR-A1 example is not an isolated case as second-genefor example, has acknowledged the deployment of autonomous sentry robots in Syria (Khurana 2018. The future of war, beginning with the trend of aucontrolled, and automated than everbefore
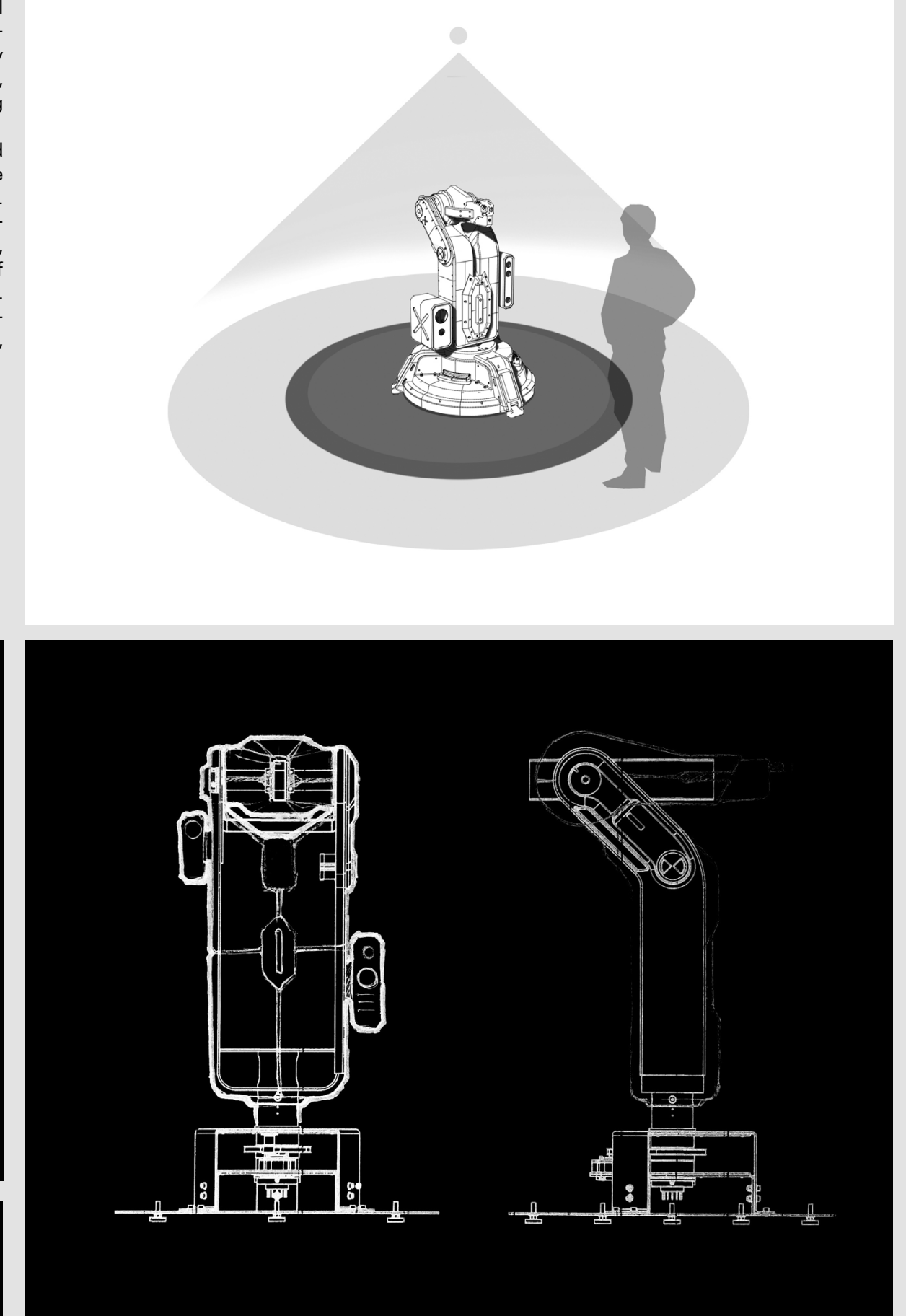

Death Inc., reflects on the impersonalization of the decision to end a human life and the role design replica inspired by the Samsung SGR-A1 model. The experience makes visitors feel as if they are the

machine's potential target, vulnerable and at the mercy of an algorithm.
When a person comes into contact with Death Inc., the machine, previously at rest, raises its robotized radius of action. Visitors can move around the weapon freely, trying to throw it off course with feints or changes of direction, but, thanks to an overhead camera hanging from the ceiling, the weapon w
never lose sight of its target.
This interactive design engenders a sort of violence
towards visitors that they try to avoid. The idea behind this behavior is to make visitors feel the their lives in the hands of an algorithm. interaction towards any type of movement is possiof being able to turn infinitely on head, the cables that control and feed these elschanics of the machine which mustresist wear from hundreds of daily interactions, can only be formed by an internal aluminum skeletonmade of tubes and 列 was the formal definition of the weapon. LAWs, due the number of cameras they require to visually recognize heir target, look simlarin appearance to part of an exhibition For this reason, and with the aim of provoking a greater impact on visitors, a or cor cover $3 \mathrm{D}$ printing was used with technology

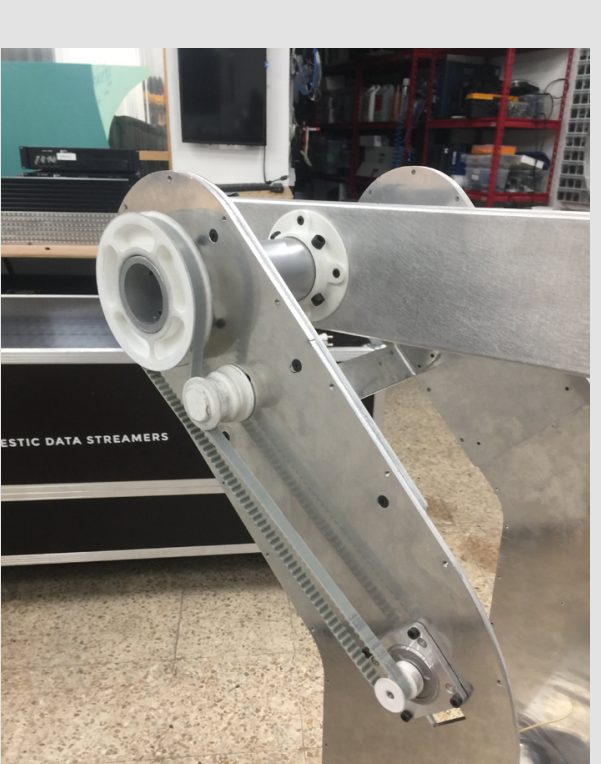

The piece uses computer vision technology (speThe piece uses computer vision technology (spe-
cifically the Python OpenCV library), with the aim of detecting the maximum number of people around the installation. The tracking system, which
uses captured images as an input through an RGB camera located behind the weapon, separates the background from the human figure and maintains its position relative to the person. The weapon not move its aim away from visitors until they ext meters) at which point it changes and ains ato new visitor.

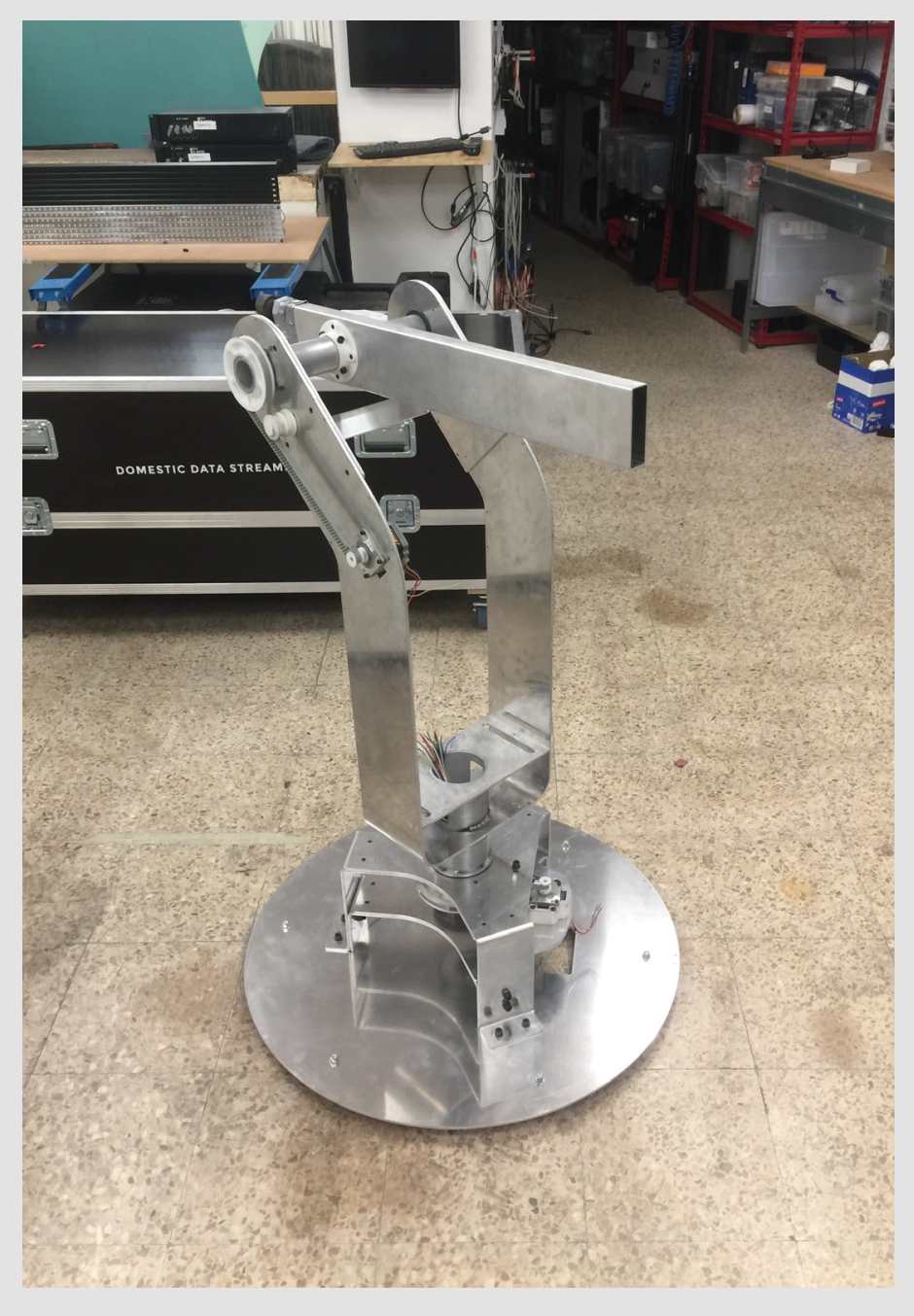


ALEX BORDANOVA

Vomestic Cata Streamers
Va estudiar Enginyeria de So Histotria a la Universitat de
Valècia

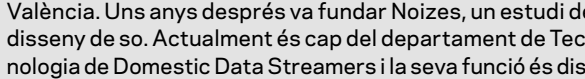
nologia de Domestic Data Streamers i la seva funcióés dis-
senyar l'arquuitectural logica itècnica de tots els projectes

paraules clau Visió Artificii
mes Letals.

Domestic Data Strem

Valencia. Unos años más tarde fundó Noizess, un estudu

de diseño de sonido. Actualmente es Director de Tecnologia en Domestic Data Streamersy
diseñar la arquitectura logica
de Domestic Data Streamers.

PALABras CLAVE Visión Artific
mas Letales.

\section{Traducció al Català}

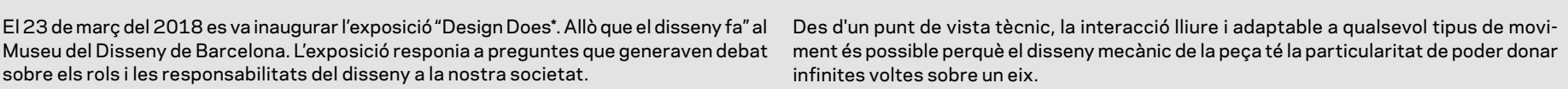
lantejava preguntes obertes, que no tenien una resposta correc

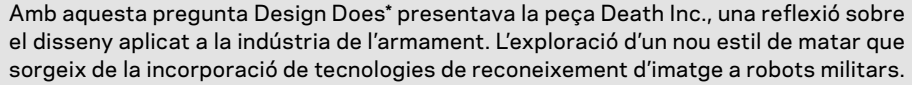

El projecte Death Inc. neix de la investigació cientifica "Autonomous Weapon Systems:
Failing the Principle of Discrimination" (Guersenzvaig 2018) que explora les consequèèSmmorals ietiques de les armes autonnomes. S'hi esmenta el cas del model SGR-A1

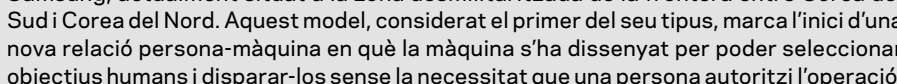
Les armes autònomes sense control humà (Humans Out-Of-The-Loop Weapons en anglès
han arribat per quedar-se. Malgrat el compromis oficialitzat per futureofifife. org contral

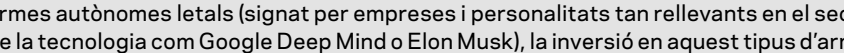

Les armes autònomes, tal com van fer en el seu dial la pólvorai ila bomba atòmica, revolu-
cionaran la nostra manera d'entendre les guerres. A dia d'avui, ''xememple de 'SGRR-A1 no

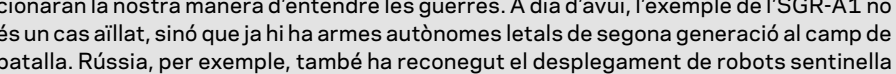
utònoms a Siria (Khurana

El futur de la guerra, que comença amb la te
tàctic, precis, controlati automàtic que mai.

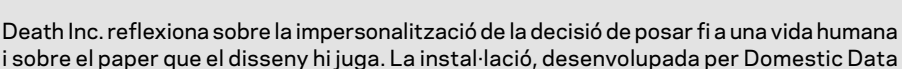
Streamers, se centra en la la interacació delis visititants amb una re replica inspiriada en el mod model

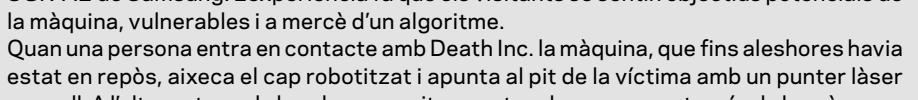

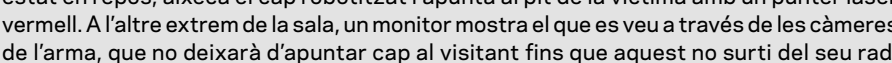

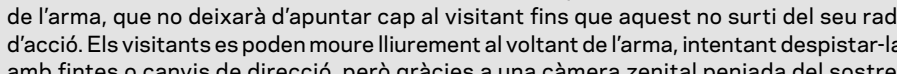
amb fintes o canvis de direccio, pero grácies a una cammera zenital penjada del sost violència que lusuari intenta evitar, però la idea darrere d'aquest comportament és fer
que senti la tensió de serv vist com un possible enemic, de sentir que la seva própia vida

TEMES DE DISSENY \#35 infinites voltes sobre un eix.
Tenint en compte la presencia de sensors, motors, càmeresi ilasers situats al cap de
l'arma els cables que controlen ialimenten aquests elements també han de poder girar

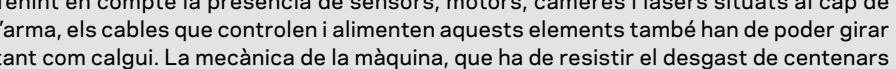
d'interaccions diàries, només pot estar formada aer un esquelet intern d'aluminin fet de
tubs i planxes plegades, tan lleugeres com resistents. Un dels reptes més complexos que vahaver de resoldre el disseny va ser la definició for-
mal del les armes. A causa de la quantititat de càmeres que necessiten per poder reconenixer

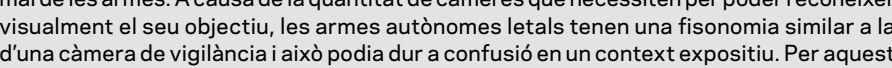

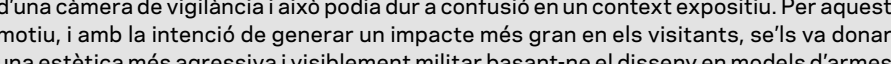

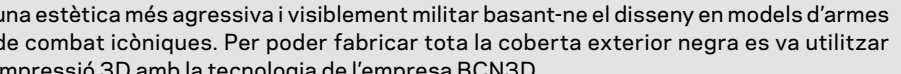
pega fa ús de tecnologia de visió artificial (concretament la biblioteca OpenCV de

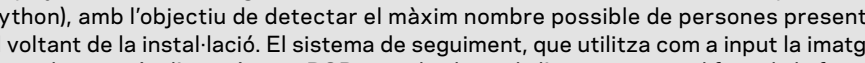

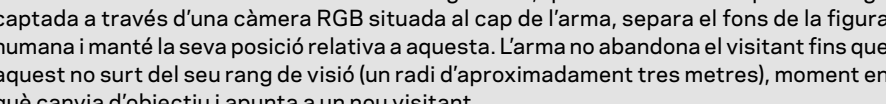

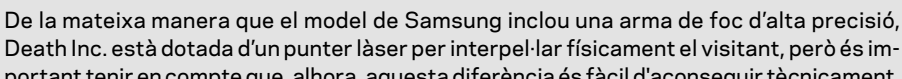
El veritable repte humanitari a què ens enfrontem és ser conscients que la tecnologia afecta la nostra moralitat i el nostre comportament, i que pot situar-nos en un terreny Death Inc. és un projecte de disseny interactiu pensat per fer reflexionar sobre la reslecnologia provocai continuara provocaar do
positiui ide vegades en un sentit negatiu.

\section{REFERÈNCIES}

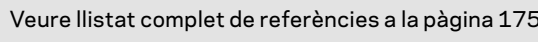

PICTORIAL

\section{Traducción al Castellano}

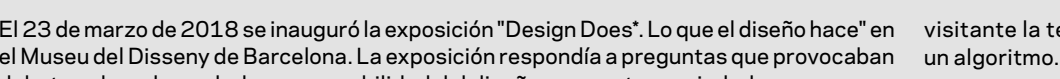
debate sobre el papel $y$ l responsabilidad del diseño en nuestra sociedad. preguntas como: ¿ ¿Debemos as automatizarlo todo?

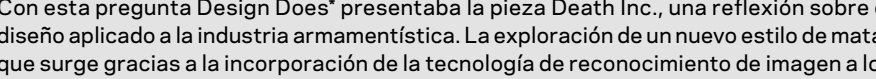
bots miltares. La muerte de un ser humano a manos reor decisión de una máauina.

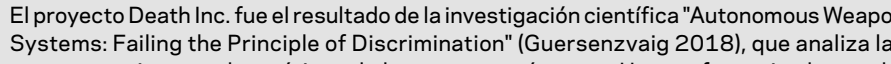

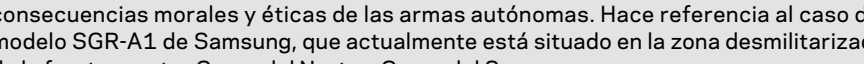
dela frontera entre Corea dei I Norte y Corea del St

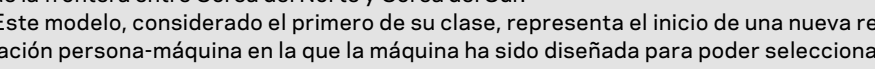
objetivos humanonos y dispararles sin que sea necesaria la autorización de una persona
para la operación.

Las armas autónomas sin control humano (Humans Out-of-the-Loop Weapons en inglés
han venido para quedarse. A pesar del compromiso oficializado por futurelife.org contra las armas autónomas letales (firmado por empressas yersonalidades tan destacadasen
el sector de la tecnologia como Google Deep Mind o Elon Musk las Sinversiones en este Las armas autónomas, como hicieron en el pasado la pólvora y la bomba atómica, revo-
lucionarán nuestra manera de entender las guerras. Actualmente, el $S \mathrm{~S} R-\mathrm{A} 1$ no oes ejemplo aislado, porque ya hay armas autónomas letales de segunda generación en el
campo de batalla. Rusia, por ejemplo, ha admitido haber desplegado robots vigía autó-
nomos en Sirial Khurana 2018).

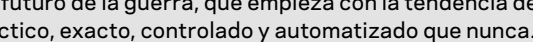

Death Inc. reflexiona sobre la impersonalización de la decisión de poner fin a una vida

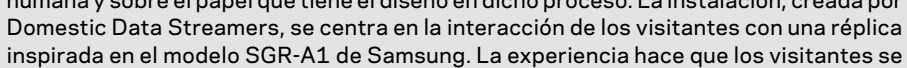
inspirada en el modelo SCRR-A1 de Samsung, La experiencia hace que los visitantes se
sientan objetivos potenciales de la máquina, vulnerables y a merced de un algoritmo. Cuando una persona entra en contacto con Death Inc. I a máquina, que hasta entonces estaba en reposo, levanta su cabezar robotizada y apunta al pecho de lavictima con un
puntero láser rojo. En el otro extremo de la sala una pantalla muestra lo que ven las cá
maras del arma, que no deja de apuntar al visititante hasta que este abandona suradio de a de ella con amagoso o cambios de dirección, pero gracias a una cámara cenitial colocada
en el techo, el arma nuncapierde de vistasu objetiviv. Este diseño de interacción genera
una esspecie de violencia que el usuario intenta evitar, y con ello se quiere transmitir al

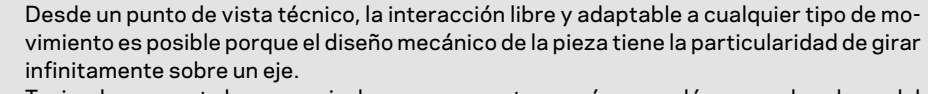

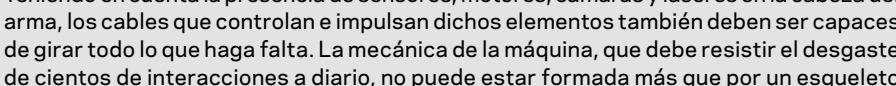

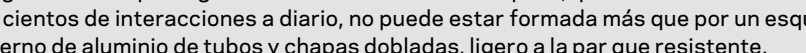

Uno de los desafios más complejos que tuvo que resolver el diseño fuu la definición formal
del arma. A causa de la cantidad de cámaras que necessitan para reconocer visualmente

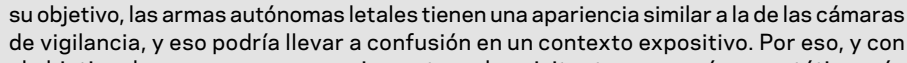
objetivo de provocar un mayor impacto en los visitantes, se creó una esteticica más

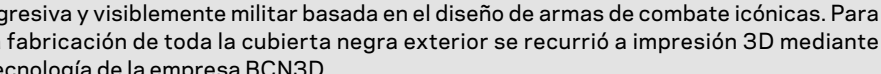

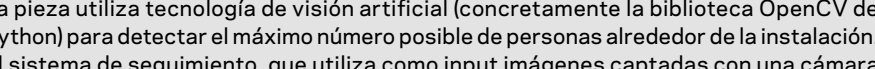
Es sistema de seguimiento, que utiliza como input imágenes captadas con una cámara
GBB situada en la cabeza del arma, separa el fondo de la figura humana y mantiene su

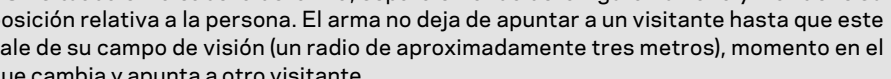
Del mismo modo que el modelo de Samsung incluye una arma de fuego dealta precición,
Death Inc. está equipada con un puntero láser yera interpelar fisicamente al visitante, Elverdadero desafio humanitario al

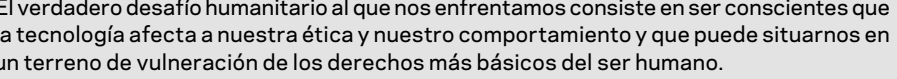
Death Inc. es un proyecto de diseño interactivo pensado para hacer reflexionar sobre
aresponsabilidadad de los diseñandores sobret todolo lo que diseñan $y$ también sobre los para bien y a vecess para mal

REFERENCIAS

P. TRIAS / A. BORDANOVA 